\title{
The (cost)-effectiveness of self-treatment of exacerbations on the severity of exacerbations in COPD patients: the COPE-II study
}

\author{
Tanja Effing ${ }^{1,2}$, Huib Kerstjens ${ }^{3}$, Paul Van Der Valk ${ }^{1}$, Gerhard Zielhuis ${ }^{4}$, Job Van Der Palen ${ }^{1,5}$ \\ ${ }^{1}$ Medisch Spectrum Twente, Department Of Pulmonology, Enschede, The Netherlands \\ ${ }^{2}$ Repatriation General Hospital, Department Of Respiratory Medicine, Daw Park, South \\ Australia, Australia \\ ${ }^{3}$ University Medical Center Groningen, And University Of Groningen, Department Of \\ Pulmonology, Groningen, The Netherlands \\ ${ }^{4}$ Radboud University Nijmegen, Department Of Epidemiology, Biostatistics, And Hta, \\ Nijmegen, The Netherlands \\ ${ }^{5}$ Department Of Research Methodology, Measurement And Data Analysis, University Of \\ Twente, Enschede, The Netherlands
}

\section{Corresponding author}

Tanja Effing

Repatriation General Hospital

Department of Respiratory Medicine

Daws Road

Fax: +61 882751195

Daw Park SA 5041

Tel: +61882751189

Australia

E-mail: tanja.effing@health.sa.gov.au

Key words: COPD Exacerbations; Clinical Epidemiology; Health Economist.

"The Corresponding Author has the right to grant on behalf of all authors and does grant on behalf of all authors, an exclusive licence (or non exclusive for government employees) on a worldwide basis to the BMJ Publishing Group Ltd and its licensees to permit this article (if accepted) to be published in [THORAX] editions and any other BMJPG Ltd products to exploit all subsidiary rights, as set out in our licence (http://thorax.bmj.com/ifora/licence.pdf). 


\section{ABSTRACT}

COPD is a chronic disease with high prevalence and quickly increasing incidence rates. We evaluated the effect of self-treatment of COPD exacerbations on the severity of exacerbations during one year. In addition, a cost-effectiveness analysis was performed.

Patients were randomly allocated to four two-hour self-management sessions, with or without training in self-treatment of exacerbations. Patients in the self-treatment group received an action plan with the possibility to initiate a prednisolone course (with or without antibiotics). During follow up all participants kept a daily symptom diary. These provided the data to calculate the frequency of exacerbations, the number of exacerbation days and mean daily severity scores.

Data were analysed of 142 randomised patients (self-treatment: $n=70$; control: $n=72$ ). The frequency of exacerbations was identical in both study groups (mean 3.5 (SD 2.7)).

Patients in the self-treatment group reported fewer exacerbation days (median self-treatment: 31(interquartile range (IQR): 8.9-67.5); control: 40 (IQR: 13.3-88.2); $p=0.064$ ), the difference was significant in the group of patients with a high number of exacerbation days per year $(>137$ (=90th percentile of the whole study population); $p=0.028)$. The mean severity score of an exacerbation day was equal in both groups. No between-group differences were found in health related quality of life. Cost-effectiveness analyses showed that applying self-treatment saved $€ 154$ per patient, with a trend towards a lower probability for hospitalisations (number/patient/year: self-treatment: 0.20 versus control: $0.33(\mathrm{p}=0.388)$ ) and a significant reduction of health care contacts (number/patient/year: self-treatment: 5.37 versus control: $6.51(\mathrm{p}=0.043))$.

We conclude that self-treatment of exacerbations incorporated in a self-management programme leads to less exacerbation days and lower costs. 


\section{INTRODUCTION}

The course of chronic obstructive pulmonary disease (COPD) is characterised by episodes of acute deterioration in respiratory health, termed 'exacerbations'. These exacerbations account for much of the morbidity, mortality, and hospital admissions in COPD patients (1). The majority of all costs in COPD patients are related to the treatment of acute exacerbations, with the costs of hospitalisations being the major component (2).

Patients seem to be capable of identifying warning signs and symptoms of exacerbations (3), but they often do not report an exacerbation to their physician and are therefore potentially delaying treatment $(1 ; 4)$. Proper guidelines for self-treatment of exacerbations might help to induce prompt treatment of exacerbations, thereby accelerating recovery and probably reducing the risk of hospitalisation. Therefore, self-treatment of exacerbations has the potential to reduce their severity and associated costs.

Until today, there is no consensus about the exact definition of a COPD exacerbation let alone its severity. Several frequently quoted definitions of exacerbations are based on respiratory symptoms $(1 ; 5)$, and therefore the number and length of exacerbations can be defined by using daily symptom scores (1). Severity of exacerbations is often characterized by type of health care consumption (e.g., courses of steroids, hospitalisations) (6), but the number of exacerbation days in combination with daily symptom scores as a measure of severity, might be a more precise alternative. Because in most COPD patients the presence of symptoms is more the rule than the exception, using the change in level of symptoms relative to baseline instead of the absolute level could increase the accuracy of the outcome measurement even more (4).

We evaluated the effects of self-treatment of exacerbations within a self-management programme in a randomised study: the COPE-II study. The severity of exacerbations (total number of exacerbation days combined with the average daily severity score) was assessed by determining change relative to baseline in daily symptoms during a one-year follow-up. In addition a cost-effectiveness analysis was performed.

\section{MATERIAL AND METHODS}

\section{Patients}

From November 2004 through July 2006, 159 patients were recruited from the outpatient department of pulmonary medicine of medisch spectrum twente hospital at enschede, the netherlands. Patients had to meet the following criteria; 1) a clinical diagnosis of COPD according to the GOLD criteria (6);2) no exacerbation in the month prior to enrolment; 3 ) $\geq 3$ exacerbations, defined as respiratory problems that required a course of oral corticosteroids and/or antibiotics, or $\geq 1$ hospitalisation for respiratory problems in the two years preceding study entry; 4) (ex) smoker; 5) age 40-75 years; 6) post-bronchodilator FEV1 25\%-80\% of predicted; 7) able to understand and read dutch; and 8) written informed consent from the subject prior to participation. Patients were excluded if they had: 1) serious other disease with a low survival rate; 2) other diseases influencing bronchial symptoms and/or lung function (e.g., cardiac insufficiency, sarcoidosis); 3) severe psychiatric illness; 4) uncontrolled diabetes mellitus during a COPD exacerbation in the past or a hospitalisation for diabetes mellitus in the two years preceding the study; 4$)$ need for regular oxygen therapy ( $>16$ hours per day or po $2<7.2 \mathrm{kpa}$ ); 5) maintenance therapy with antibiotics; ; 6) known $\alpha 1$-antitrypsine deficiency; 7) disorders or progressive disease seriously influencing walking ability (e.g., amputation, paralysis, progressive muscle disease). The hospital's medical ethics committee approved the study. 


\section{Study design}

A two-by-two factorial design was used, meaning that two independent interventions (selftreatment and a community-based physiotherapeutic exercise programme (COPE-active)) were evaluated using one design (table 1). The effect of a self-management programme including self-treatment of exacerbations (self-treatment group) was compared with a selfmanagement programme only (control group). Patients receiving COPE-active were divided equally over the self-treatment and control group using a minimisation program (7), minimising differences between groups in gender, current smoking, FEV1 predicted $(\leq$ or $>$ $50 \%$ ), and use of inhaled corticosteroids. This design assumes that both interventions do not interact with each other which need to be verified before the final analyses. Measurements of health related quality of life, anxiety and depression, and lung function were performed at baseline, and after 7 and 12 months.

Table 1. Description of the design of the cope-ii study, including the two interventions (self-treatment of exacerbations and cope-active) and the measurements at baseline, 7, and 12 months

\begin{tabular}{llccccc}
\hline & & \multicolumn{2}{c}{$\begin{array}{l}\text { Self-treatment group } \\
\text { (=intervention group) }\end{array}$} & \multicolumn{2}{c}{ Control group } \\
\cline { 3 - 6 } $\begin{array}{l}\text { Time } \\
\text { schedule }\end{array}$ & & Subgroup 1 & Subgroup 2 & Subgroup 3 & Subgroup 4 \\
\cline { 3 - 7 } & Interventions & & $\mathrm{X}$ & $\mathrm{X}$ & $\mathrm{X}$ & $\mathrm{X}$ \\
\hline Month 0 & Baseline measurement & $\mathrm{X}$ & $\mathrm{X}$ & $\mathrm{X}$ & $\mathrm{X}$ \\
Month 1 & Self-management courses & $\mathrm{X}$ & $\mathrm{X}$ & - & - \\
Month 1-12 & Self-treatment of exacerbations & $\mathrm{X}$ & - & $\mathrm{X}$ & - \\
Month 2-7 & Cope-active: 'compulsory' & $\mathrm{X}$ & $\mathrm{X}$ & $\mathrm{X}$ & $\mathrm{X}$ \\
Month 7 & Measurement & $\mathrm{X}$ & - & $\mathrm{X}$ & - \\
Month 8-12 & Cope-active: 'voluntary' & $\mathrm{X}$ & $\mathrm{X}$ & $\mathrm{X}$ & $\mathrm{X}$ \\
Month 12 & Measurement & & & & \\
\hline
\end{tabular}

\section{Self-management sessions and self-treatment intervention}

Smokers were offered a three month smoking cessation program prior to the group-allocation (8). After randomisation, patients of both study groups and their partners were offered four weekly two-hour small-group (approximately 5 patients) self-management sessions given by a respiratory nurse and a physiotherapist. The intention of the courses was to change the patients' disease behaviour by increasing their knowledge, confronting them with consequences of specific behaviour, and supplying them with tools to deal with different components of their disease. Patients of the intervention group additionally received training in self-treatment of exacerbations. Four, 13, and 26 weeks after the last course, the respiratory nurse contacted all patients by phone to recall the items addressed during the self-management courses. Patients were supplied with a booklet with the content of the courses.

All patients were educated in completing daily diaries, in which they had to report whether their major symptoms (breathlessness, sputum production, sputum colour) and minor symptoms (cough, wheeze, running nose, sore throat, and fever $\left(>38.5^{\circ} \mathrm{c}\right)$ ) according to anthonisen (5) were beyond normal. At inclusion all patients received a 'what is normal' card, which described their individual levels of major symptoms in stable health state. When patients experienced no deterioration of any of the symptoms listed on the diary, they could tick 'no change in symptoms'. In other cases, they had to report on all the symptoms in the diary whether the level of each symptom was normal, slightly increased, or clearly increased.

The start of the exacerbation was defined as 'a clear negative change in two major 
symptoms or one major and one minor symptom from baseline, for at least two consecutive days'. The day that the exacerbation was resolved was defined as the first day of: 1) three successive days that the patient had returned to his normal health state; or 2) seven consecutive days on which patients continuously reported no or only a slight increase in symptoms compared to baseline, with no fever or change in sputum colour.

Patients in the self-treatment group were educated in early recognition of exacerbations and were taught when to start a course of oral prednisolone by using an action plan that was linked to the daily diary. For treatment of an exacerbation, either diagnosed by the physician or by the patient from the action plan, a course of prednisolone (30 $\mathrm{mg}$ for seven days) was the standard medication. Change of sputum colour was the indicator to combine prednisolone with antibiotics (amoxicillin/clavulanic acid 500/125 $\mathrm{mg}$ [augmentin ${ }^{\circledR}$ ] three times daily for a 7 days, or (in case of hypersensitivity) doxycycline $100 \mathrm{mg}$ daily for 10 days.

Patients in the control group were instructed to call the study office if they felt in need of assistance for any worsening of their respiratory symptoms, which normally would have prompted them to call their chest physician or general practitioner instead. A consultation with a chest physician was scheduled within 12 hours.

\section{Outcomes}

The total number of exacerbation days was calculated by summing up all exacerbation days per patient per year, based on the information from the diaries. The severity of an exacerbation day was calculated with help of symptom scores. The major symptoms were scored as: normal $=0$; small increase $=1$; or clear increase $=2$. The minor symptoms were scored $0,0.5$ and 1 , respectively. Sputum colour was scored as: normal $=0$, different from normal $=2$; and fever: no $=0$, yes $=1$. Adding all these scores resulted in a daily score with a range from 0-11 points. When patients were admitted to the hospital a daily score of 15 points was assigned. If patients had less than 4 months of complete diary data, they were excluded from the analyses.

Health related quality of life (HRQoL) was measured with the self-administered Chronic Respiratory Questionnaire Standardised (CRQ-SAS) (9). The questionnaire captures four domains: dyspnoea, fatigue, emotional functioning, and mastery. A change of 0.5 in a domain is considered to be clinically relevant at the individual level (10). Health status was evaluated by the self-administrated Clinical COPD Questionnaire (CCQ) (11). A change of 0.4 represents the minimal important difference for an individual patient (12). Anxiety and depression was measured with the Hospital Anxiety and Depression Scale (HADS) (13). This instrument produces separate scores for anxiety and depression ranging from 0-21. A score over 10 is judged to be a predictor of a clinical diagnosis of anxiety and depression. Finally, information about hospital admissions, emergency room visits, scheduled and emergency outpatients visits, phone calls with physicians, consultations with the general practitioner, and information about medication use was collected for cost-effectiveness analyses.

\section{Statistical analyses}

We calculated that 79 patients per treatment group were required to detect a difference of 2 exacerbation days (SD 4.5 days) with $80 \%$ power and a two-sided $0.05 \alpha$-level test. All statistical analyses were performed on an intention-to treat basis using SPSS version 12.0. Between-group differences of 'exacerbation days/year' and 'mean severity score/year' were assessed by comparing the proportion of patients with an individual value larger than the median, the $75^{\text {th }}$, and the $90^{\text {th }}$ percentile. Subsequently chi-square statistics for categorical variables were applied. Between-group differences of continuous variables were assessed by 
analysis of repeated measurements using the spss mixed models procedure, and in case of continuous variables (number of hospitalisations, courses of prednisolone / antibiotics) by the student T-test (normal distribution) or the wilcoxon rank sum test (non-normal distribution). In case of a non-normal distribution, the median combined with the interquartile range (IQR) was presented.

\section{Cost-effectiveness}

A decision analytical model with a time perspective of 12 months was developed to evaluate the cost effectiveness of a self-management programme including self-treatment of exacerbations versus a self-management programme only. Resource use was multiplied by 2004 unit prices (14). Medication costs were based on market prices and included a $€ 6$ dispensing fee and 6\% VAT for each prescription. We took the perspective of the health care payer. The costs-effectiveness ratio was calculated as costs per exacerbation day prevented, per hospital admission prevented, and per health care contact prevented.

For sensitivity analysis a Monte Carlo simulation with 1000 iterations was performed to evaluate the relative impact of likely variations in the parameters in the decision analytical model. Therefore, cost parameters and probabilities were varied simultaneously over their ranges and associated 95\% CI. For the costs of health care contacts and hospital admissions a normal distribution was used, while for the costs of the self-management programme, exacerbations, and regular medication a triangular distribution was used. Logistic normal distributions were used for all probabilities.

\section{Results}

Of the 421 eligible patients, 80 were assigned to the self-treatment group and 79 to the control group (figure 1). Between inclusion and the baseline measurements three patients dropped out in each group and insufficient diary data were delivered by 11 patients. Baseline characteristics of the remaining 142 patients are presented in table 2 and were similar between both groups with respect to all measured prognostic factors. The drop outs $(n=11)$ did not differ from the remaining group with respect to all factors, except the medical research council dyspnoea scale (drop outs versus remaining patients: 2.3 (SD 1.1) versus 3.0 (SD 1.1; $\mathrm{p}=0.041$ ). During the one-year follow-up, three self-treatment group patients dropped out, of which one died due to an intracerebral haemorrhage. Thus, 67 patients in the self-treatment group and 72 patients in the control group completed the one-year follow-up.

Table 2. Baseline characteristics of the two treatment groups

\begin{tabular}{lll}
\hline Number of patients & $\begin{array}{l}\text { Self-treatment } \\
(\mathrm{n}=70)\end{array}$ & $\begin{array}{l}\text { Control } \\
(\mathrm{n}=72)\end{array}$ \\
\hline Age (mean \pm sd; years) & $63.1 \pm 7.9$ & $63.7 \pm 8.0$ \\
Male (\%) & 57.1 & 61.1 \\
Smokers (\%) & 32.9 & 33.3 \\
Medical research council dyspnoea scale (mean $\pm \mathrm{sd})$ & $2.31 \pm 1.06$ & $2.33 \pm 1.14$ \\
Lung function post bronchodilation (mean $\pm \mathrm{sd})$ & & \\
$\quad$ FEV1 (l) & $1.44 \pm 0.56$ & $1.41 \pm 0.50$ \\
$\quad$ FEV1 \% predicted value & $50.7 \pm 16.3$ & $49.6 \pm 15.3$ \\
$\quad$ VC (l) & $3.64 \pm 1.08$ & $3.66 \pm 0.85$ \\
Inhaled corticosteroid use (\%) & 88.6 & 84.7 \\
\hline
\end{tabular}


The frequency of exacerbations was the same in both study groups (3.5 (SD2.7)). Figure 2 shows that patients in the self-treatment group had a lower total number of exacerbation days than patients in the control group. Differences were significant in the group patients with a relatively high number of exacerbation days per year $(>137$ days/year $(=\mathrm{P} 90$ of the whole study population); $p=0.028$ ). The mean severity score of an exacerbation day did not differ between both study groups (self-treatment: 5.1 (SD 2.4); control: 5.1 (SD 3.0)). Additional analyses of the more severe exacerbation days (daily score $>6$ points, which represents a maximum score on all major symptoms), showed a (non significant) trend towards a higher number of severe exacerbation days/year in the control group (median self-treatment: 11(IQR: 0.8-21.0); control: 15 (IQR: 3.3-26.8); $\mathrm{p}=0.124$ ).

The self-reported number of prednisolone (median: self-treatment 2.6 (IQR: 1.0-5.0); control 1.7 (IQR: 1.0-3.2); $\mathrm{p}=0.055$ ) and antibiotic courses (median: self-treatment 2.0 (IQR: 0.8-4.0); control 1.1 (IQR: 0.0-2.9); $\mathrm{p}=0.048$ ) were higher in the self-treatment group. The number of hospital admissions was 14 in the STG and 24 in the control group $(\mathrm{p}=0.388)$. No between-group differences were found in the CRQ-components, the CCQ (sub)-scores, the HAD scores, and fev $1 \%$ of predicted over the 12-month period.

\section{Cost-effectiveness}

Table 3 presents the percentages, which are derived from the COPE-II study, for each step in the decision analytic model (figure 3). Figure 3 shows, besides the percentages of patients for each step, also the percentages of patients in the different branches. For example, the percentage of patients in the self-treatment group having at least one exacerbation (90\%), one health care contact (100\%), and one hospitalisation $(17.7 \%)$ is $15.9 \%(90 \% * 100 \% * 17.7 \%)$. The costs made by a patient in this branch are $€ 6388$. These costs consists of: the costs of a self-management course including self-treatment (€118), regular medication costs (€761), health care contacts costs (€281) (table 4), and the hospitalisation costs (€5227). In this branch medication for an exacerbation is incorporated in hospitalisation costs. The percentage of patients in each branch will finally determine the weight of the branch costs to the total costs / patient / study group (e.g., 15.9\%* €6388).

Table 3. Base case values of percentages of patients with a copd exacerbation, a hospitalisation and medication use for an exacerbation

\begin{tabular}{lll}
\hline & Base case value (95\% CI) \\
\hline Patients with an exacerbation (\%) & $90.0(83.1$ to 96.9$)$ & Control \\
$\begin{array}{l}\text { Patients with a hospitalisation when having } \\
\text { an exacerbation and health care contact (\%) }\end{array}$ & $17.7(8.8$ to 26.6$)$ & $24.6(14.7$ to 34.5) \\
$\begin{array}{l}\text { Patients with medication use for an } \\
\text { exacerbation when not hospitalised but }\end{array}$ & $90.8(84.0$ to 97.6$)$ & $61.4(50.2$ to 72.6$)$ \\
$\begin{array}{l}\text { having an exacerbation and a health care } \\
\text { contact (\%) }\end{array}$ & & \\
\hline
\end{tabular}


Table 4. Mean costs $(€)$ per patient per year in patients with and without self-treatment using 2004 costs prices

\begin{tabular}{llll}
\hline Costs per patient $(€)$ & $\begin{array}{l}\text { Self-treatment } \\
(\mathrm{n}=70)\end{array}$ & $\begin{array}{l}\text { Control } \\
(\mathrm{n}=72)\end{array}$ & Difference \\
\hline Self-management courses & 117.68 & 111.60 & 6.08 \\
Regular medication $^{*}$ & 761.21 & 644.00 & 117.21 \\
Health care contacts $^{\dagger}$ & 262.49 & 366.70 & -104.21 \\
patients with an exacerbation $_{\text {patients without an exacerbation }}$ & $280.52(90 \%)$ & $402.94(84.7 \%)$ & \\
Hospitalisation costs $^{\dagger}$ & $100.21(10 \%)$ & $166.07(15.3 \%)$ & \\
Medication for exacerbations $^{* *}$ & 823.24 & 1020.36 & -197.12 \\
\hline & 75.30 & 51.40 & 23.90 \\
\hline & & & \\
Total costs $(€)$ & 2039.92 & 2194.06 & -154.14 \\
\end{tabular}

*Includes $€ 6$ pharmacy cost and 6\% vat per prescription

${ }^{\dagger}$ Includes (telephone) consultations with the physician and general practitioner, and emergency room visits

$\$$ Costs per hospitalisation day: $€ 337$ (includes medication costs); mean of 12.21 and 9.08 hospitalisation days per admission and 14 and 24 admissions for respectively the selftreatment and control group. The sum of $€ 5227$ for hospitalisation costs as mentioned in an example in the text are the hospitalisation costs per self-treatment patient having at least one hospitalisation: average costs of a hospitalisation * (total number of hospitalisations in the self-treatment group / total number of intervention patients with $>$ one exacerbation).

${ }^{* *}$ Mean of medically treated exacerbations per patient per year for respectively the selftreatment and control group: treatment with prednisolone and antibiotics: 2.31 versus 1.43 (€ 27,69 per exacerbation); treatment with solely prednisolone: 0.93 versus 0.82 (€7.79 per exacerbation); and treatment with solely antibiotics: 0.21 versus $0.27(€ 19.90$ per exacerbation)

The 12 month costs data are presented in table 4 and the effect data in table 5. The selftreatment strategy was dominant over the control strategy. Patient in the self-treatment group generated $€ 2040$ in direct medical costs versus $€ 2194$ in the control group. So, applying selftreatment saved $€ 154$ per patient. The number needed to treat (NNT) to prevent one hospitalisation was 7.4, and the NNT to prevent one health care contact was 0.9 . The results of the sensitivity analysis with regard to costs for hospital admissions and health care contacts are shown in figure 4 the dominant outcome: a positive treatment effect in combination with saving money was seen in the majority of the outcomes (hospitalisations: $63.3 \%$; health care contacts: $49.2 \%$ ). Positive effects, independent of costs, were seen in $95.1 \%$ and $74.4 \%$ of the iterations for hospitalisations and health care contacts, respectively.

Table 5 Effects per patient per year of copd patients receiving a self-management programme with and without self-treatment

\begin{tabular}{lllr}
\hline Effect per patient & $\begin{array}{l}\text { Self-treatment } \\
(\mathbf{n = 7 0 )}\end{array}$ & $\begin{array}{l}\text { Control } \\
(\mathbf{n = 7 2 )}\end{array}$ & Difference \\
\hline Number of exacerbation days & 42.20 & 64.50 & -22.30 \\
Number of hospitalisations $_{\text {Number of health care contacts }}^{*}$ & 0.200 & 0.334 & -0.134 \\
\hline
\end{tabular}


* Includes (telephone) consultations with the physician and general practitioner, and emergency room visits

\section{Discussion}

This study showed that self-treatment of exacerbations leads to fewer exacerbation days in combination with an increase in the use of courses of prednisolone and antibiotics. No between-group differences in the number of exacerbations, average daily severity score, and hrqol were found. Cost-effectiveness analyses showed that the self-treatment strategy was dominant over (i.e. Compared favourably with) the control strategy with lower probabilities for hospitalisations and health care contacts in the self-treatment group, combined with cost savings.

Prior to the study we hypothesised that the severity of exacerbations, defined by the length of the exacerbation in combination with the daily severity scores, would be influenced by the self-treatment guidelines because self-treatment would lead to a more rapid start of treatment after the early recognition of an exacerbation. A priori, we did not expect an effect on exacerbation frequency because self-treatment was initiated after the onset of an exacerbation. Both hypotheses were confirmed by our results. Our study data showed a reduction of the number of exacerbation days in the self-treatment group. Remarkably this was not accompanied by a reduction in the mean daily severity score. A closer look suggests that this lack of difference in daily severity score between both study groups is, at least partly, explained by the fact that self-treatment particularly reduces the number of the less severe exacerbation days (towards the resolution phase of the exacerbation) in the self-treatment group. As a consequence, the average daily severity score (total severity score / number of exacerbation days / patient) of the remaining days of the self-treatment group goes up. We hypothesise that the mean severity score in the control group was higher due to the fact that patients in this group experienced both a higher number of more severe exacerbation days as well as a higher number of less severe exacerbation days. This hypothesis is supported by the (non-significant) trend towards a larger number of more severe exacerbation days (symptom score $>6$ points) in the control group.

The difference in severity of exacerbations between both study groups might have been underestimated. By participating in the self-management programme, patients of the control group might have become more aware of the negative consequences of exacerbations and might have contacted the hospital quicker than they were used to. Furthermore, because of their participation in this study, control patients experienced a very low threshold for contacting, and a very short delay in actually being seen by a chest physician ( $<12$ hours), relative to usual care practice. This all might have led to a more rapid start of treatment and as a consequence a less severe exacerbation.

All patients, so also the control group, received a self-management programme. This increased the costs also in the control group, and raises the question whether a control group without self-management programme would have constituted a clearer design. However, a comparison between our self-treatment group and a control group receiving no intervention at all would probably have led to similar conclusions with regard to cost-effectiveness, since the favourable effects of the intervention would probably have increased further. Furthermore, the costs of the self-management programme $(€ 112)$ were very low and avoiding them would therefore have saved little.

SImilar to other studies (15), including COPE-I (16), our data showed a significantly higher reported use of courses prednisolone and antibiotics in the self-treatment group. But since the number of reported courses in the self-treatment group was still lower than the actual number of exacerbations reported in the diaries, meaning that prednisolone was not used 
during each exacerbation, we are convinced that the between-group difference in courses is due to a underreporting of respiratory complaints to the chest physician by patients in the control group, rather than a reflection of overtreatment in the self-treatment group. This underreporting has also been observed in several other studies $(1 ; 3 ; 17)$.

No effects were found on HRQoL and lung function. Associations between exacerbation frequency and HRQoL $(1 ; 18 ; 19)$ and between severity of exacerbations and HRQoL (18) have been demonstrated by others. Since we found no effect on exacerbation frequency, a relation with changes in hrqol or lung function was no longer expected.

In this study, change from baseline was used to define symptoms and therefore exacerbation severity. This method was introduced because almost all COPD patients always experience at least some symptoms in stable state. Using health care consumption (courses of prednisolone / antibiotics) instead of this change from baseline in symptoms, leads to a lower number of exacerbations in both study groups. On the other hand, using symptoms from the diaries, but only as presence or absence (instead of change relative to baseline) would most likely lead to the detection of more (and more severe) exacerbations. Whether the use of these other definitions would also affect the estimates of treatment effects remains to be studied.

Using a 2-by-2 factorial design confers the theoretical risk of interaction between both interventions. A priori, there were no reasons to assume an interaction of a physiotherapeutic exercise program and self-treatment of exacerbations with regard to the different outcomes. Since the proportion of patients receiving physiotherapy was the same in both study groups, possible (additional) effects of the COPE-active program will have affected both study groups and will thereby not directly influence the between-group differences. Before the final analysis we have checked for interaction between both interventions and we concluded that interaction was not present.

The dominance of the self-treatment strategy was largely caused by the lower probability for hospitalisation in the self-treatment group. As expected, no significant between-group differences in hospital admissions were found, since this study was not at all powered to detect a difference in hospital admissions.

In summary, we conclude that self-treatment incorporated in a self-management programme in COPD patients leads to less exacerbation days at lower costs, while not leading to overtreatment. Given the rise in prevalence of COPD, and the costs associated with treatment, we suggest to start implementation programmes incorporating careful instructions on self-treatment as a part of self-management programmes.

\section{Acknowledgements}

We gratefully acknowledge all the members of our department of pulmonary medicine, but especially the nurse practitioner clara van ommeren, the lung function technician irma wissink, the data managers betty rinsma and petra meerlo, and of course the patients.

This study was funded by a grant from the dutch asthma foundation.

\section{Disclosure of interests:}

The authors te, hk, pv, gz and jp have declared no conflict of interest including involvement with any organisation with a direct financial, intellectual, or other interest in the subject of the manuscript (e.g. An author is an employee, consultant, shareholder, or paid expert witness of the organisation). 
Legend Figure 2:

* Chi-square test $(<$ or $>35$ (=median of the whole population): $\mathrm{p}=0.06$

$\dagger \quad$ Chi-square test $(<$ or $>69.4$ ( $=\mathrm{p} 75$ of the whole population): $\mathrm{p}=0.38$

$\$$ Chi-square test $(<$ or $>137.2$ ( $=$ p90 of the whole population): $\mathrm{p}=0.03$

Reference List

(1) Seemungal TA, Donaldson GC, Paul EA, Bestall JC, Jeffries DJ, Wedzicha JA. Effect of exacerbation on quality of life in patients with chronic obstructive pulmonary disease. Am J Respir Crit Care Med 1998 may;157(5 pt 1):1418-22.

(2) Rutten-Van Molken MP, Postma MJ, Joore Ma, van genugten Ml, leidl R, jager JC. Current and future medical costs of asthma and chronic obstructive pulmonary disease in the netherlands. Respir Med 1999 nov;93(11):779-87.

(3) Kessler R, Stahl E, Vogelmeier C, Haughney J, Trudeau E, Lofdahl CG, et al. Patient understanding, detection, and experience of COPD exacerbations: an observational, interview-based study. Chest 2006 jul;130(1):133-42.

(4) Wilkinson TM, Donaldson GC, Hurst JR, Seemungal TA, Wedzicha JA. Early therapy improves outcomes of exacerbations of chronic obstructive pulmonary disease. Am J Respir Crit Care Med 2004 jun 15;169(12):1298-303.

(5) Anthonisen NR, Manfreda J, Warren CP, Hershfield ES, Harding GK, Nelson NA. Antibiotic therapy in exacerbations of chronic obstructive pulmonary disease. Ann Intern Med 1987 feb;106(2):196-204.

(6) Rabe KF, Hurd S, Anzueto A, Barnes PJ, Buist SA, Calverley P, et al. Global strategy for the diagnosis, management, and prevention of chronic obstructive pulmonary disease: gold executive summary. Am J Respir Crit Care Med 2007 sep 15;176(6):532-55.

(7) Altman DG, Bland JM. Treatment allocation by minimisation. Bmj 2005 apr 9;330(7495):843.

(8) Pieterse ME, Seydel ER, Devries H, Mudde AN, Kok GJ. Effectiveness of a minimal contact smoking cessation program for dutch general practitioners: a randomized controlled trial. Prev med 2001 feb;32(2):182-90.

(9) Puhan MA, Behnke M, Laschke M, Lichtenschopf A, brandli o, Guyatt GH, et al. Self-administration and standardisation of the chronic respiratory questionnaire: a randomised trial in three german-speaking countries. Respir Med 2004 apr;98(4):34250 .

(10) Juniper EF, Guyatt GH, Willan A, Griffith LE. Determining a minimal important change in a disease-specific quality of life questionnaire. J Clin Epidemiol 1994 jan;47(1):81-7.

(11) Van der Molen t, Willemse BW, Schokker S, Ten Hacken NH, Postma DS, juniper ef. Development, validity and responsiveness of the clinical COPD questionnaire. Health Qual Life Outcomes 2003 apr 28;1(1):13.

(12) Kocks JW, Tuinenga MG, Uil SM, van Den Berg JW, Stahl E, van der Molen T. Health status measurement in COPD: the minimal clinically important difference of the clinical COPD questionnaire. Respir res 2006;7:62.

(13) Zigmond AS, Snaith RP. The hospital anxiety and depression scale. Acta Psychiatr Scand 1983;67:361-70. 
(14) Oostenbrink JB, Bouwmans CAM, Koopmanschap MA, Rutten FFH. Handleiding voor kostenonderzoek. College Voor Zorgverzekeringen; 2004.

(15) Turnock AC, Walters EH, Walters JA, Wood-Baker R. Action plans for chronic obstructive pulmonary disease. Cochrane Database Syst Rev 2005;(4):cd005074.

(16) Monninkhof E, van Der Valk P, van Der Palen J, van Herwaarden C, Zielhuis G. Effects of a comprehensive self-management programme in patients with chronic obstructive pulmonary disease. Eur Respir J 2003 nov;22(5):815-20.

(17) Langsetmo L, Platt RW, Ernst P, Bourbeau J. Underreporting exacerbation of chronic obstructive pulmonary disease in a longitudinal cohort. Am j Respir Crit Care Med 2008 feb 15;177(4):396-401.

(18) Miravitlles M, Ferrer M, Pont A, Zalacain R, Varez-Sala JL, Masa F, et al. Effect of exacerbations on quality of life in patients with chronic obstructive pulmonary disease: a 2 year follow up study. Thorax 2004 may;59(5):387-95.

(19) Spencer S, Jones PW. Time course of recovery of health status following an infective exacerbation of chronic bronchitis. Thorax 2003 jul;58(7):589-93.

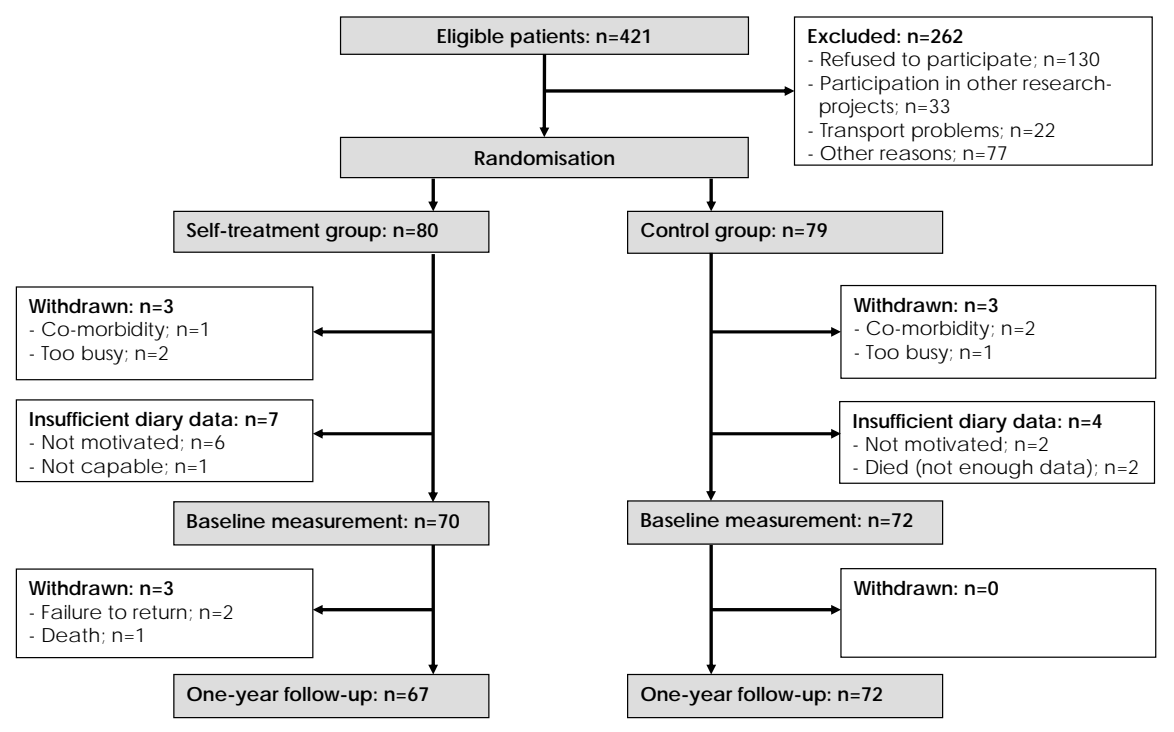

Figure 1. Flow diagram of subject progress through the COPE-II study 


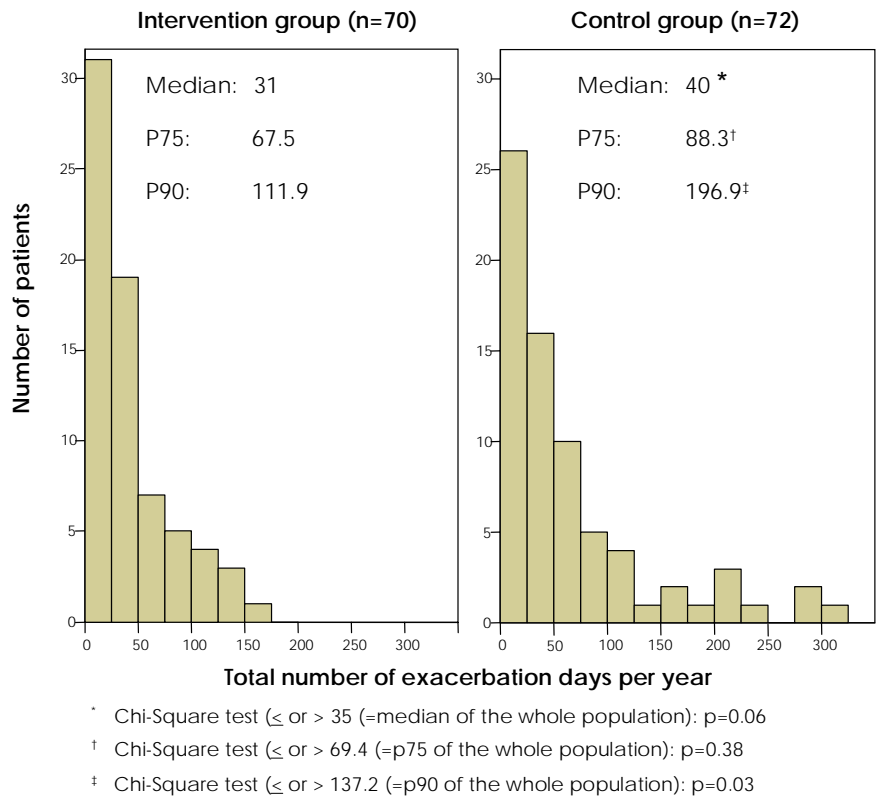

Figure 2. Number of exacerbation days per year for patients in the intervention and control group; median, $75^{\text {th }}$ and $90^{\text {th }}$ percentile of both study groups and the corresponding $p$-valuesfor group differences are also reflected 


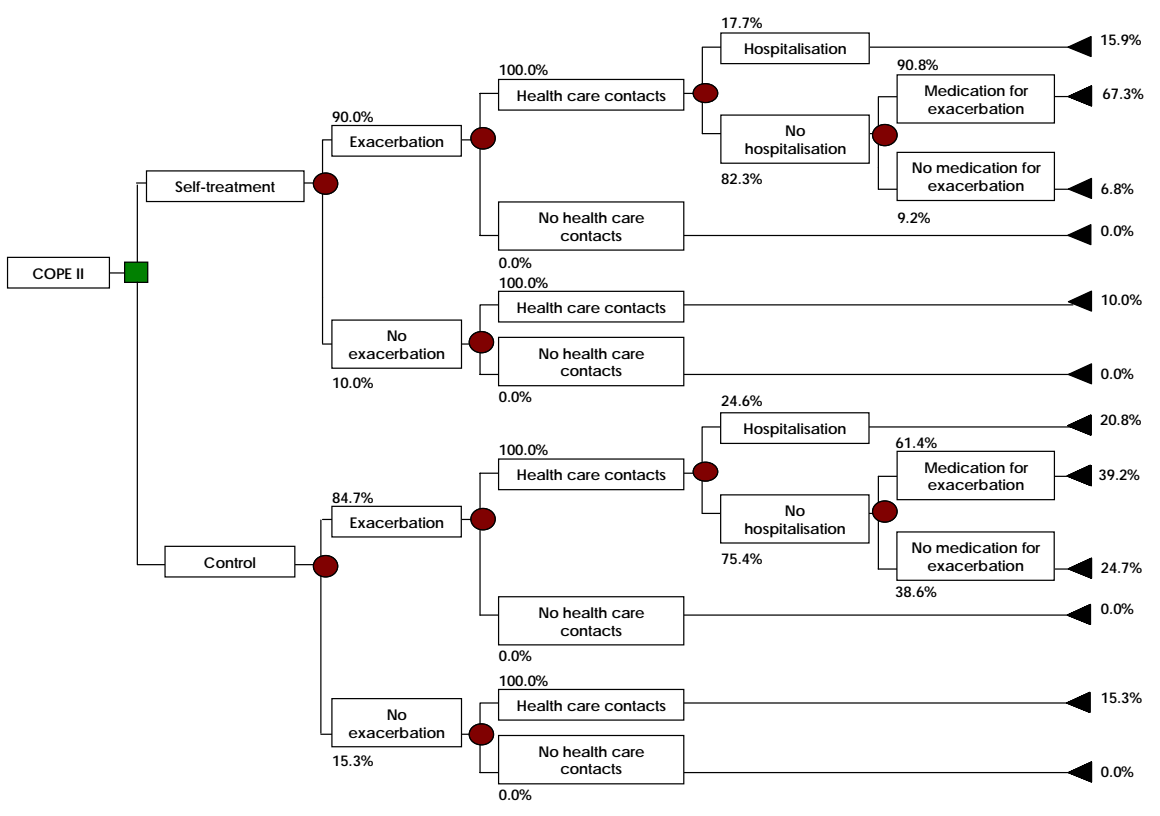

Figure 3. Decision analytic model with percentages of patients with exacerbations, health care contacts, hospitalisations and medication for exacerbations for the self-treatment and control group 

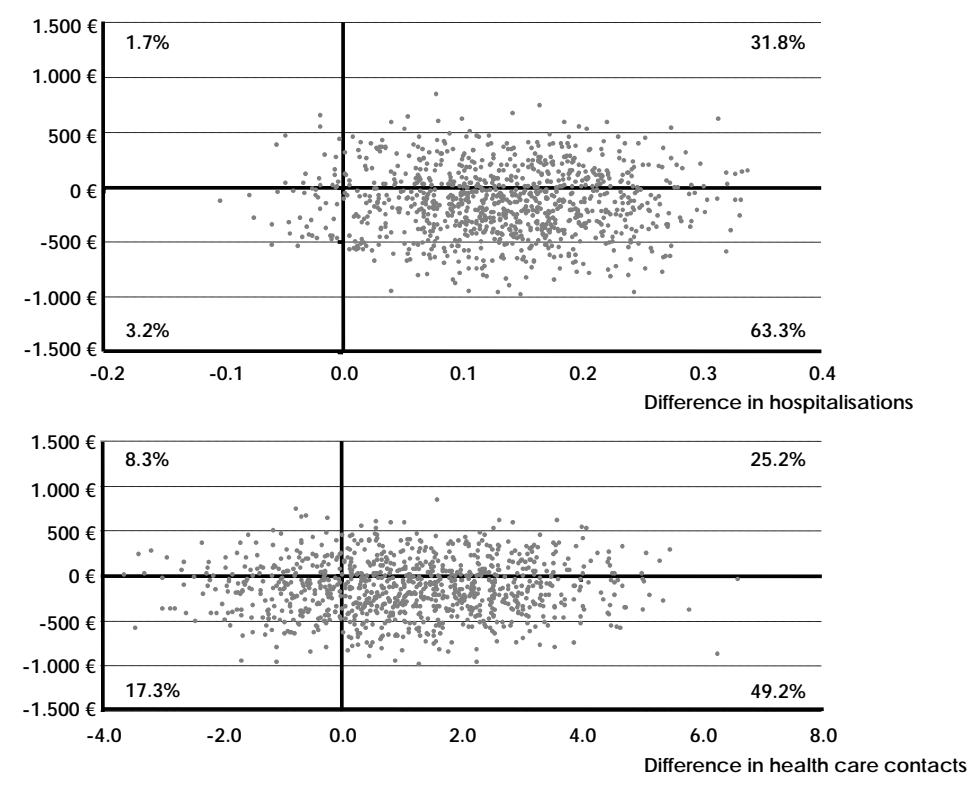

Figure 4. Results of the sensitivity analysis (1000 iterations) on cost per hospital admission prevented and costs per health care contact prevented. A negative $€$ amount and a positive difference in the hospitalisations and health care contacts favours the self-treatment intervention. In addition the percentages of outcomes in every quadrant are given. 